\title{
COMPARISON OF ACCURACY OF EXPECTED DATE OF DELIVERY CALCULATED BY NAEGLE'S RULE AND BY ULTRASOUND SCAN
}

Dileep Javadekar, R. P. Patange, Vaishali Whaval, Archana Rokade

1. Assistant Professor. Department of Obstetrics \& Gynaecology, Krishna Institute of Medical Sciences, Karad. Maharashtra.

2. Professor \& HOD. Department of Obstetrics \& Gynaecology, Krishna Institute of Medical Sciences, Karad. Maharashtra.

3. Assistant Professor. Department of Obstetrics \& Gynaecology, Krishna Institute of Medical Sciences, Karad. Maharashtra.

4. Assistant Professor. Department of Obstetrics \& Gynaecology, Krishna Institute of Medical Sciences, Karad. Maharashtra.

\section{CORRESPONDING AUTHOR:}

Dr. Dileep Javadekar,

Alchemy, Vishrambag,

Sangli- 416415.

E-mail: dileep2355@yahoo.com.

ABSTRACT: The expected date of delivery is of paramount importance to pregnant woman, her relatives, and treating doctor. It is useful for arranging help required and to take appropriate obstetric decisions. This study was aimed to find more accurate method to know Expected date of delivery. This was prospective study done on 800 new cases coming to Antenatal Outpatient clinic. A total of 318 cases were included in analysis. Rest of them were excluded for various reasons. In the patients selected Expected date was calculated according to Naegle's rule calculation (L.M.P. date +280days) and by measuring femur length and biparietal diameter of fetal head measured on ultrasound scan and using Hadlock charts already available on ultrasound machines. Actual date when these patients went in labour spontaneously was noted eventually. Accuracy rates for ultrasound scan and menstrual period estimates of date of delivery were calculated as the percentage estimates accurate to within a given number of days. Significance tests and confidence intervals for differences in these paired rates were calculated.

The cases where the estimated dates of delivery disagree are termed discrepant. For 33 patients the estimated dates of delivery from the two methods were identical and in 27 of these cases they were exactly correct. The percentages of the 285 discrepant cases in which the scan gave closer prediction of the date of delivery than the last menstrual period.

It can be concluded that all persons involved with antenatal care, may be doctors or the patients and relatives should rely the estimated date of delivery derived from ultrasound scan and ignore the one calculated by Naegle's rule once the satisfactory scan has been obtained.

The study was undertaken to compare and know the more accurate and reliable method to calculate Expected Date of Delivery for the pregnant women coming to Antenatal Outpatient department. Total 800 women were included in the study initially coming from different parts of the district. Out of the above only 318 women were taken for analysis and the rest were excluded for various reasons.

KEYWORDS: gestational age; Expected date of delivery; ultrasound scan; Last menstrual period.

INTRODUCTION: The Expected date of delivery has remained always of profound importance for the treating doctor as well as the expectant woman and her relatives. From medical point 
view it is very much important for the well being of the mother and child as placental aging may cause serious damage to the baby in uterus after that date. For patient and relatives the importance cannot be overemphasized. They have to arrange for the help, for the transport vehicle, for the leave sanction from the work place and what not. Its accuracy is therefore of paramount importance. With the widespread availability of ultrasound scanning and the development of standard fetal measurement, ${ }^{1,2}$ most women now have two independently derived estimates which may differ : a calculation based on last menstrual period (L.M.P.+280 days) and a prediction based on the measurement by ultrasound scanning .

The doctors, nurses, and patient with relatives are always confused about which date is to be referred as the expected date of delivery. A small number of patients do not remember the last menstrual period date and for them the dilemma does not exist. They have to go according to the scan report and the confusions is over for them; but when the patient gives the exact history of last menstrual period and the details of usual cycle length the confusion exists. The aim of this study was to determine which method is the more accurate predictor of the date of delivery.

METHOD: Total 800 patients attending antenatal clinic were randomly selected for this study who came in their first half of gestational period (approximately 20 weeks). Thorough history was taken as usual. The Expected date of delivery was calculated by Naegle's rule. (Add 9 months and 7 days to last menstrual period.)

Ultrasound scanning was performed at around 17-18 weeks' gestation in usual manner. It was for gross fetal anomaly if any and fetal biometry. After the ultrasound scan had been performed the measurements of biparietal diameter and femur length were recorded. Campbell and Obrien had prepared Referral Graphs for ready reference of gestational age ${ }^{3,4}$. Instead the ultrasound machines were preprogrammed by Hadlock charts and automatically gave expected date of delivery and corresponding weeks of gestation. The machine calculated expected date of delivery was used in our study. If there was a small discrepancy between the two the mean was taken and if the discrepancy was greater than one week the case was excluded from the study as it could suggest a fetal abnormality.

After delivery, details of the pregnancy outcome were recorded.

Following cases were excluded from analysis:

1. Patients whose pregnancy was terminated spontaneously or deliberately for obstetric reason before 28 weeks of gestation.

2. Those whose labour was induced.

3. Those with abnormal baby or multiple pregnancy.

4. Those who did not come for delivery in our institute.

5. Those who had to be taken for Caesarian Section before labour established.

6. Those who were not sure about their Last menstrual period date.

7. Those with very irregular menstrual periods prior to conception.

STATISTICAL ANALYSIS: Accuracy rates for ultrasound scan and menstrual period estimates of date of delivery were calculated as the percentage estimates accurate to within a given number of days. Significance tests and confidence intervals for differences in these paired rates were calculated using exact binomial method ${ }^{5}$ (Appendix 1). The choice of sample size was guided by the finding of Campbell and colleagues that the date of delivery estimated from measurement of 
biparietal diameter at 12-16 weeks' gestation was not accurate to plus or minus 14 days of the actual date in $10.6 \%$ of 1678 patients 6 .

RESULTS: A total of 800 women presented for antenatal booking during 6 months period. They were from rural and semirural area and from all different casts and religions.

Four hundred and eighty two women were excluded from the analysis for following reasons:

1. Patients whose pregnancy was terminated spontaneously or deliberately for obstetric reason before 28 weeks of gestation...35

2. Those whose labour was induced....106

3. Those with abnormal baby or multiple pregnancy....17

4. Those who did not come for delivery in our institute....108

5. Those who had to be taken for Caesarian Section before labour established....42

6. Those who were not sure about their Last menstrual period date...106

7. Those with very irregular menstrual periods prior to conception....68

The remaining 318 women were included in the analysis. They had a mean age of 26 years (range 19-33 years) and were scanned at a mean gestation of 19 weeks(17-24 weeks).

One hundred and eleven women were primigravidas, 124 second gravida while 83 were $3^{\text {rd }}$ gravid or above.

Graph 1 shows the accuracy rates of the last menstrual period and scan dates as estimates of the actual date of delivery, An error of zero days means that the scan or the last menstrual period predicted the actual date of delivery exactly and an error of 14 days means that the scan or the last menstrual period estimate is 14 days earlier or later than the actual date of delivery. The accuracy rate of the scan was greater than that of the menstrual period method for errors of 12 days or less, the same for 13 day error and similar for 14 day error.

The cases where the estimated dates of delivery disagree are termed discrepant. For 33 patients the estimated dates of delivery from the two methods were identical and in 27 of these cases they were exactly correct. The percentages of the 285 discrepant cases in which the scan gave closer prediction of the date of delivery than the last menstrual period are shown in Table 1. The proportion of cases where the scan was the better predictor was never less than $50 \%$ and became significantly greater than $50 \%(p<0.05)$ for discrepancies of five days or more.

DISCUSSION: A study from a London hospital accepting many tertiary referrals from district general hospital obstetric units and serving a multiracial local population showed that biparietal diameter measurements performed at between 12 and 18 weeks' gestation were significantly more accurate predictors of the actual date of delivery that last menstrual period for all 4527 women examined, including those who were sure of the date of their last menstrual period. ${ }^{6}$ It is said that as exact prediction of expected date of delivery is not possible by any method it should be replaced by probable week of delivery, so that the anxiety of patient and relatives will be alleviated 7 . But in our class of patients we have seen that they are not satisfied at all by such substitution. After every scan almost upto 38 to 40 weeks of gestation they continue to ask about expected date of delivery.

As a result of this work many obstetricians now prefer the scan estimated date of delivery in all cases, but some still take the last menstrual period estimated date of delivery if the two predictors differ by only a few days ${ }^{8}$. A study from Edinburgh analysed the certainty of last menstrual period dates in details ${ }^{9}$. Even for those with the greatest certainty of menstrual history from three groups of women, a scan estimated date of delivery derived from crown 
rump length or biparietal diameter was more accurate than last menstrual period estimated date of delivery.

A hospital based study from Oklahoma reached a conflicting conclusion ${ }^{10}$. The authors suggested that gestational age based on good menstrual records supported by a pelvic examination in the first trimester may be more reliable than even the best ultrasound method for dating. They say that if the woman is very sure about her last menstrual period date the estimated date of delivery by Naegle's calculation should not be changed unless the discrepancy between last menstrual period and scan estimated date of delivery is 14 days or more.

The British Medical Ultrasound Society's view is that if the scan estimated date of delivery differs from the clinical, including last menstrual period, assessment by more than one week, then the ultrasonic assessment should be the working gestational age and the clinical assessment should be discarded 11 .

Once a woman has had a scan at hospital with a reliable biparietal diameter and femur length measurement, the estimated date of delivery calculated by sonologists should always be taken in preference to the last menstrual period prediction, even when the woman is absolutely sure of her dates and however little the discrepancy between the two predictions. It is always good to repeat scan if there is large discrepancy keeping in mind possibility of fetal abnormality or intrauterine growth retardation.

\section{Appendix 1.}

TABLE 1 Comparison of accuracy rates.

Consider the following table, which gives a breakdown of the accuracy (to within seven days or less) of the estimated date of delivery according to the two methods:

\begin{tabular}{|l|l|l|l|}
\hline & \multicolumn{3}{|l|}{ Percentage of women(n=318) } \\
\hline & $\begin{array}{l}\text { LMP } \\
\text { estimate } \\
\text { accurate }\end{array}$ & $\begin{array}{l}\text { LMP estimate } \\
\text { inaccurate }\end{array}$ & Total \\
\hline Scan estimate accurate & 39.6 & 26.4 & 66 \\
\hline $\begin{array}{l}\text { Scan estimate } \\
\text { inaccurate }\end{array}$ & 12.3 & 21.7 & 34 \\
\hline Total & 51.9 & 48.1 & 100 \\
\hline n= total number of women. LMP = Last menstrual period \\
\hline
\end{tabular}

The difference in accuracy rates (scan minus last menstrual period) is $66.0 \%$ minus $51.9 \%$ which equals $14.1 \%$. However, each of these rates includes 126 patients $(39.6 \%)$ for whom both methods were accurate. The rates are therefore correlated and the standard test of difference between independent proportions is inapplicable. Instead, the difference may be seen to be mathematically identical to the difference between rate A (scan accurate, last menstrual period inaccurate, 26.4\%) and rate B (scan inaccurate, last menstrual period accurate, 12.3\%). Rates A and B are calculated from non-overlapping subsets of patients and so may be presumed to be statistically independent. If rate $A$ equals rate $B$, both estimates are equally accurate. $A$ test of rate $A$ equals rate $B$, both estimates are equally accurate. A test of rate $A$ equals rate $B$ is the same as attest of rate $A /(A+B)=1 / 2$ and may be based on the binomial distribution with proportion $1 / 2$. Similarly, confidence intervals for rate A minus rate B (and by implication for the difference in accuracy rates, which is identical to rate A minus rate B) may be found using the binomial distribution. In the above example, rate A minus rate B (14.1) is significantly from zero 
$(p<0.05)$, so the scan is significantly better than the last menstrual period method for predicting the date of delivery to within one week of the actual date. The confidence interval for rate $\mathrm{A}$ minus rate $\mathrm{B}$ is $1.5 \%$ to $24.7 \%$.

TABLE 2

Percentage of Discrepant cases where scan gave closer prediction

\begin{tabular}{|l|l|}
\hline $\begin{array}{l}\text { Discrepancy in estimated date of delivery } \\
\text { (days) }\end{array}$ & $\begin{array}{l}\% \text { of discrepant cases where scan more } \\
\text { accurate than LMP (95\% confidence interval) }\end{array}$ \\
\hline$>=1(n=285)$ & $56(45$ to 66$)$ \\
\hline$>=2(n=246)$ & $56(39$ to 61$)$ \\
\hline$>=3(n=207)$ & $58(46$ to 70$)$ \\
\hline$>=4(n=180)$ & $60(47$ to 72$)$ \\
\hline$>=5(n=159)$ & $66(52$ to 79$)$ \\
\hline$>=6(n=135)$ & $67(51$ to 80$)$ \\
\hline$>=7(n=117)$ & $69(52$ to 83$)$ \\
\hline$>=8(n=87)$ & $76(57$ to 90$)$ \\
\hline$>=9(n=75)$ & $76(55$ to 91$)$ \\
\hline$>=10(n=69)$ & $78(56$ to 93$)$ \\
\hline$>=11(n=60)$ & $75(51$ to 91$)$ \\
\hline$>=12(n=54)$ & $78(52$ to 94$)$ \\
\hline$>=13(n=42)$ & $86(57$ to 98$)$ \\
\hline$>=14(n=30)$ & $90(56$ to 100$)$ \\
\hline$>=15(n=30)$ & $90(56$ to 100$)$ \\
\hline$n=n u m b e r$ of discrepant cases & \multicolumn{1}{|l}{} \\
\hline LMP $=$ Last menstrual period &
\end{tabular}




\section{Graph 1 : Accuracy of estimated date of delivery for differing errors from the actual date of delivery}

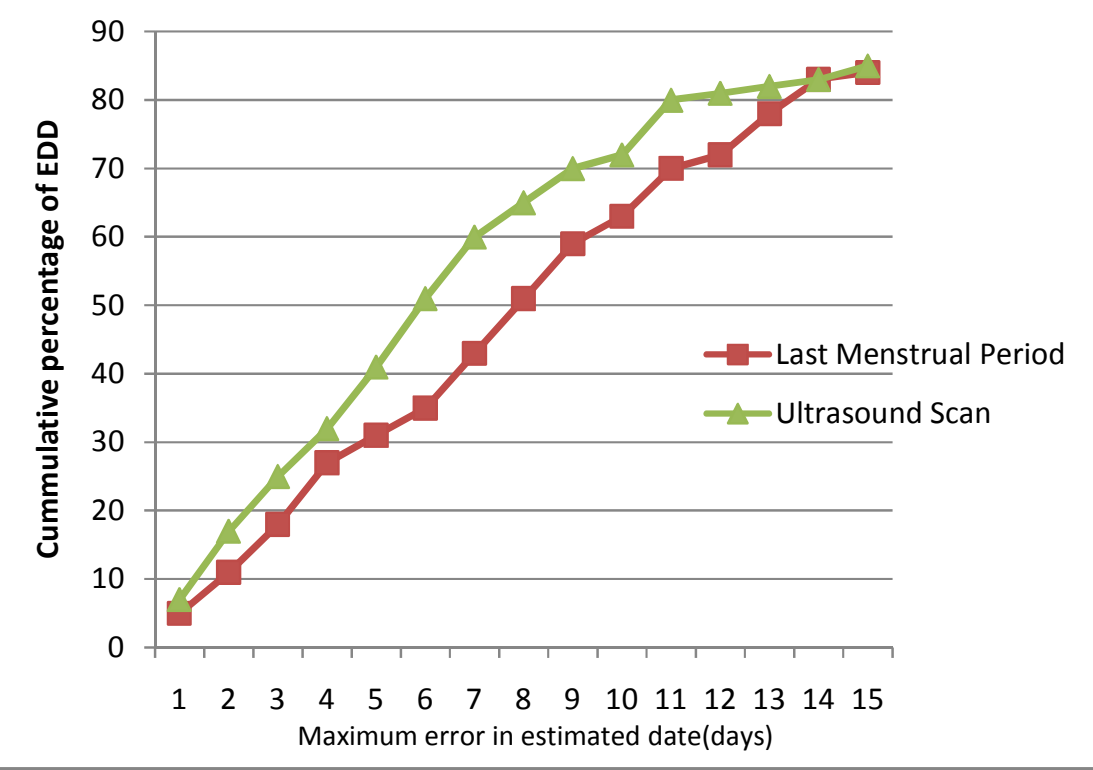

$\mathrm{EDD}=$ Estimated date of delivery

\section{REFERENCES:}

1. Campbell S. An improved method of fetal cephalometry by ultrasound. J.Obstet $\mathrm{Br}$ Cmwlth 1968; 568576

2. Queenan JT, O'Brien GD, Campbell S. Ultrasound measurement of fetal limb bones. Am J Obstet Gynecol 1980; 138: 297-302

3. Campbell S, Newman GB. Growth of the fetal biparietal diameter during normal pregnancy. J Obstet Gynecol Br Cmwlth 1971; 78: 513-519.

4. O'Brien GD, Queenan JT. Growth of the ultrasound fetal femur length during normal pregnancy. Am J Obstet Gynecol 1981; 141: 833-837.

5. Armitage P, Berry G. Statistical methods in medical research. Oxford: Blackwell, 1987:123.

6. Campbell S, Warsof SL, Little D, Cooper DJ. Routine ultrasound screening for the prediction of gestational age. Obstet Gynecol 1985; 65: 613-620.

7. Saunders N, Paterson C. Can we abandon Naegele's rule? Lancet 1991; 337:600-601.

8. Hall MH. Definitions used in relation to gestational age. Paediatr Perinatal Epidemiol 1990; 4: 123-128.

9. Geirsson RT, Busby-Earle RMC. Certain dates may not provide a reliable estimate of gestational age. Br J Obstet Gynecol 1991; 98:108-109.

10. Rossavik IK, Fishburne JI. Conceptional age, menstrual age and ultrasound age: a second-trimester comparison of pregnancies of known conception date with pregnancies dated from the last menstrual period. Obstet Gynecol 1989; 73: 243-249.

11. British Medical Ultrasound Society Fetal Measurements Working Party. Clinical applications of ultrasonic fetal measurements. London: British Institute of Radiology, 1990. 